\title{
The Framingham cardiovascular risk score and 5-year progression of multiple sclerosis
}

Martina Petruzzo ${ }^{1 *}$, Antonio Reia ${ }^{1 *}$, Giorgia Teresa Maniscalco², Fabrizio Luiso ${ }^{1}$, Roberta Lanzillo $^{1}$, Cinzia Valeria Russo ${ }^{1}$, Antonio Carotenuto ${ }^{1}$, Lia Allegorico ${ }^{2}$, Raffaele Palladino ${ }^{3}$ Vincenzo Brescia Morra ${ }^{1}$, and Marcello Moccia ${ }^{1}$.

*Authors contributed equally as first names.

1 Multiple Sclerosis Clinical Care and Research Centre, Department of Neuroscience, Reproductive Science and Odontostomatology, Federico II University, Naples, Italy

2 Department of Neurology, Ospedale Cardarelli, Naples, Italy

3 Department of Public Health, Federico II University, Naples, Italy

\section{Correspondence to Marcello Moccia}

Multiple Sclerosis Clinical Care and Research Centre

Department of Neuroscience, Federico II University

Via Sergio Pansini 5, building 17, groundfloor

80131 Naples, Italy

E-mail: moccia.marcello@gmail.com

Tel/fax: +390817462670

Total word count: 3836

Keywords: Multiple sclerosis; cardiovascular; relapse; disability

This article has been accepted for publication and undergone full peer review but has not been through the copyediting, typesetting, pagination and proofreading process, which may lead to differences between this version and the Version of Record. Please cite this article as doi: $\underline{10.1111 / \text { ENE. } 14608}$

This article is protected by copyright. All rights reserved 
DR. MARTINA PETRUZZO (Orcid ID : 0000-0003-1372-9290)

DR. ROBERTA LANZILLO (Orcid ID : 0000-0001-6388-8180)

DR. ANTONIO CAROTENUTO (Orcid ID : 0000-0002-1574-9693)

DR. MARCELLO MOCCIA (Orcid ID : 0000-0003-2613-3090)

Article type : Original Article

\title{
The Framingham cardiovascular risk score and 5-year progression of multiple sclerosis
}

\begin{abstract}
Background. Cardiovascular risk factors and comorbidities can affect the prognosis of multiple sclerosis (MS). The Framingham risk score is an algorithm that can estimate the 10year risk of developing macrovascular disease.

Objective. To evaluate possible association between the Framingham risk score at baseline, and MS relapses, disability and disease-modifying therapy (DMT) choices over 5-year followup.

Methods. This is a retrospective cohort study including 251 MS subjects. At baseline, we calculated the Framingham risk score considering the following variables: age, sex, diabetes, smoking, systolic blood pressure, and body mass index. MS outcomes including relapses, disability and treatments were collected over 5 years. Cox proportional regression models were employed to estimate hazard ratios (HR).

Results. 1-point increase in the Framingham risk score was associated with $31 \%$ higher risk of relapse $(\mathrm{HR}=1.31 ; 95 \% \mathrm{Cl}=1.03,1.68), 19 \%$ higher risk of reaching of EDSS $6.0(\mathrm{HR}=1.19$; $95 \% \mathrm{Cl}=1.05,3.01)$, and $62 \%$ higher risk of $\mathrm{DMT}$ escalation $(\mathrm{HR}=1.62 ; 95 \% \mathrm{Cl}=1.22,3.01)$.

Conclusions. Higher cardiovascular risk was associated with higher risk of relapses, disability, and DMT escalation in MS. Early identification, correction and treatment of cardiovascular comorbidities should be carefully considered within MS management.
\end{abstract}




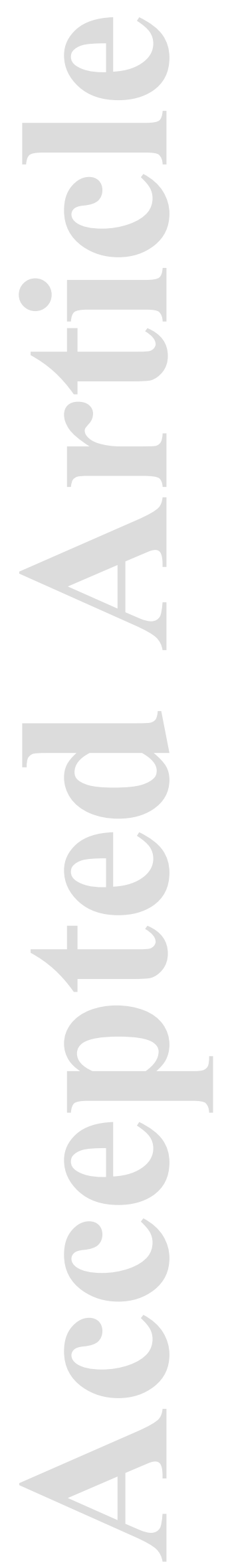

This article is protected by copyright. All rights reserved 


\section{Introduction}

Multiple Sclerosis (MS) is a chronic inflammatory and degenerative disease of the central nervous system, whose underlining causes remain unknown, though genetic and environmental factors are thought to be involved in its pathogenesis ${ }^{1}$. MS is associated with progressive disability accrual and quality of life impairment, further worsened by comorbid conditions ${ }^{2}$. The impact of cardiovascular comorbidities on prognosis of patients with MS has been examined by several studies. Overall, hypertension, ischemic heart disease, overweight, cerebral and peripheral artery disease have been associated with MS disability, relapses and clinical progression ${ }^{3-11}$. There is great attention to cardiovascular comorbidites due to their high prevalence in MS. In addition, available cardiovascular treatments, such as statins, have been associated with reduced brain atrophy and, possibly, improved long-term clinical disability in $\mathrm{MS}^{12,13}$.

In a previous study, we have already assessed the cardiovascular risk in MS using the Framingham risk score, which was associated with MS disability and clinical course (i.e., relapsing or progressive) ${ }^{4}$. The Framingham risk score predicts the percent risk of developing heart failure, coronary, cerebral or peripheral artery disease within 10 years, using age, sex, diabetes, smoking, systolic blood pressure, and BMI as input variables ${ }^{14}$. Hereby, we followed up the original study population for a five-year time (from 2014 to 2019), to assess whether increased Framingham risk score at baseline is associated with increased risk of the following MS-specific incident outcomes: (i) relapses; (ii) disability; (iii) treatment choices (i.e., escalation of disease modifying treatment (DMT)).

This article is protected by copyright. All rights reserved 


\section{Methods}

Study design and population

This is a retrospective cohort study evaluating the association between Framingham Risk Score at baseline, and MS clinical variables in a population of MS subjects over a five-year period. The present study has been conducted at the MS Clinical Care and Research Centre of the Federico II University of Naples, Italy, between September 2014 and Dicember 2019.

The study was approved by the Federico II Ethics Committee (355/19). All patients signed informed consent authorizing the use of anonymized data collected routinely as part of the clinical practice, in line with data protection regulation (GDPR EU2016/679). The study was performed in accordance with good clinical practice and Declaration of Helsinki.

Inclusion criteria were: 1) baseline assessment with Framingham risk score measurement in September 2014 as for our previous study ${ }^{4}$; 2) clinical follow-up with MS- and cardiovascular records avaible for at least one year after September 2014.

Exclusion criteria were: 1 ) age at baseline $<18$ years; 2 ) participation in clinical trials during the study period; 3) incomplete clinical records.

\section{Cardiovascular variables}

At baseline (September 2014), we computed the Framingham risk score for subjects with MS using age (at baseline), sex, diabetes (yes/no), smoking (yes/no), systolic blood pressure, and $\mathrm{BMI}^{14}$. Full details for Framingham risk score assessment have been reported elsewhere 4

During the 5-year observation, we recorded the occurrence of cardiovascular comorbidities and treatments on each visit (every three to six months), and, for the purposes of the present study, we extracted cardiovascular events which were part of the original Framingham study (hypertension, transient ischemic attack, stroke, angina, and acute myocardial infarction) ${ }^{14}$.

\section{MS clinical variables}

This article is protected by copyright. All rights reserved 
Relapses were recorded and reported on an annual basis (annualized relapse rate- ARR), and time from baseline visit to the first relapse was calculated. MS subjects were categorized according to the clinical course in relapsing remitting MS (RRMS) or secondary progressive MS (SPMS). Disability was scored with the Expanded Disability Status Scale (EDSS) by certified clinicians, and time from baseline to the reaching of EDSS 6.0 was calculated. DMTs were recorded over study duration, with some patients being not on treatment; also, during follow-up, we recorded patients with DMT escalation (e.g., from Interferon-beta, Glatiramer Acetate, Teriflunomide, or Dimethyl Fumarate to Alemtuzumab, Fingolimod, Natalizumab, Ocrelizumab, or Rituximab). This classification complies with current European Medicines Agency indications ${ }^{15}$, suggesting the use of some DMTs for escalation purposes in the presence of .

\section{Sample size}

The sample size was selected a priori based on our previous study ${ }^{4}$. Considering a normal distribution of variables to be analyzed on regression models, given a $5 \%$ minimum detectable effect size, a two-sided tail, and a 5\% $\alpha$ error, a sample of 251 patients would be able to achieve $94 \%$ power $^{16}$.

\section{Statistical analyses}

Results are presented as mean (standard deviation), number (percent), and median (range), as appropriate.

Association between Framingham risk score and ARR was evaluated using a Poisson regression model, including follow-up time as offset. Association between Framingham risk score and EDSS at follow-up was evaluated using an ordered logistic regression model. The hazard ratios for the risk of relapse, EDSS 6.0, and DMT escalation in relation to Framingham risk score were evaluated using Cox proportial hazards regression models; individuals were considered at risk for the entire study period or until reaching study endpoints and censored in case of loss at follow-up or end of study period. The assumption of parallel hazard functions over time was met. To test this assumption we examined plots of log (-log survival time) against log survival time and Schoenfeld residuals against survival time; in addition, we used linear regression of Schoenfeld residuals on time to test for independence between 
residuals and time ${ }^{17}$. These models were adjusted for age, sex, disease duration, baseline EDSS, disease subtype (RR or SP), and follow-up duration. Results are presented as coefficients (Coeff), hazard ratios (HR), 95\% Confidence Intervals $(95 \% \mathrm{Cl})$, and $\mathrm{p}$-values, as appropriate.

Statistical analyses were run using Stata 15.0. Results are considered statistically significant for $p<0.05$.

\section{Data availaibility}

Data is available upon request to the corresponding author. 


\section{Results}

Of the original population ( $n=265$ ), we were able to include 251 patients, with 14 patients being lost at follow-up (5.2\%). Baseline demographics and clinical features of included patients are reported in Table 1. We included patients with at least one year follow-up from baseline visit; follow-up was $4.6 \pm 1.0$ years (ranging from 1.1 to 5.3 years).

\section{Cardiovascular progression}

Considering average follow-up duration (4.6 years), and average Framigham risk score (6.5\%), cardiovascular events would have been expected in $3.0 \%$ of the population. During the follow-up, we recorded new onset of 1 stroke, and 4 angina, but no transient ischaemic attacks, or myocardial infarction, overall involving $5 \mathrm{MS}$ patients $(2.0 \%$ of included population).

\section{MS progression}

MS clinical features are reported in Table 2. Each additional percent Framingham risk score was associated with 40\% higher ARR ( $p<0.01$ ) (Table 2 and Figure 1a), and 30\% higher relapse risk ( $p=0.02$ ) (Table 2 and Figure 2a). Each additional percent Framingham risk score was associated with 2.7 higher EDSS at follow-up ( $p<0.01$ ) (Table 2 and Figure $\mathbf{1 b}$ ), and 20\% higher risk of reaching of EDSS $6.0(p=0.01)$ (Table 2 and Figure $2 b$ ). Full results, including also covariates, are reported in Supplementary Table 1.

DMTs are reported in Table 3. Over the follow-up, 135 patients (53.8\%) changed of DMT at least once. On average, patients received 1.8 \pm 0.9 different DMTs during follow-up. Among patients who changed of DMT, 60 (44.4\%) escalated to higher efficacy DMTs, whilst 75 (55.6\%) remained on DMTs within the same efficacy range. Each additional percent Framingham risk score was associated with $60 \%$ higher risk of DMT escalation $(p=0.03)$ (Table 2 and Figure 2c). Full results, including also covariates, are reported in Supplementary Table 1. 


\section{Discussion}

We evaluated the impact of multiple cardiovascular risk factors at baseline, combined in a percent risk score (i.e. the Framinghm risk score), on MS course over 5 years. Higher cardiovascular risk at baseline was associated with worse MS outcomes. In particular, each additional percent Framingham risk score was related to $40 \%$ higher relapse rate, $20 \%$ higher risk of reaching of EDSS 6.0, and 60\% higher risk of DMT escalation.

MS is a disabling disease with heterogeneity in clinical outcomes. Most previous natural history studies focused on demographics and MS-related clinical features, whilst comorbidity can also account for the variability of MS progression ${ }^{11}$. The impact of cardiovascular comorbidities on MS prognosis has been examined by several studies ${ }^{3}$, but we have hereby examined the combination of multiple cardiovascular risk factors alltogether.

The association between higher Framingham risk score and relapses could be mediated by a number of cardiovascular comorbidities. For instance, adverse lipid profile was associated with greater risk of active lesions on brain MRI ${ }^{18,19}$, a surrogate marker of relapses. Also, hyperlipidaemia and the coexistence of multiple comorbidities, such as hypertension and ischemic heart disease, were associated with higher relapse rate in a large Canadian sample $(n=885)^{5}$. In our previous study, Framingham risk score was not different between patients with clinical relapses in the previous 12 months ${ }^{4}$, though we have now investigated this association prospectively, over 5 years, and using both relapse risk and rate.

In line with the association between Framingham risk score and relapses, we have also found an association between higher Framingham risk score and DMT escalation. As such, patients with higher cardiovascular risk have higher probability of switching towards more active treatments. A previous Italian real-life multicenter study showed that comorbidities increased risk of DMT switch, also as a consequence of poor tolerance ${ }^{20}$, whilst, in a Canadian study, comorbidities did not seem to affect the choice of treatment ${ }^{7}$. However, these studies included a number of different comorbidities (e.g. anxiety, depression, epilepsy, migraine, other autoimmune disorders), that reduced the contribution of 
cardiovascular risk factors towards DMT choice. Overall, in our study, the increased cardiovascular risk possibly mediated higher disease activity first, and subsequent DMT escalation. Thus, modifying cardiovascular risk factors could ultimately reduce relapse risk and DMT escalation, also contributing ro reduced costs for MS management ${ }^{21}$.

We have also found an association between higher cardiovascular risk and disability progression, measured with EDSS and reaching of EDSS 6.0 (a milestone of disability, predicting the mortality in MS) ${ }^{22}$. In previous studies, diabetes, obesity, and hypertension were associated with MS-related disability, measured with EDSS and walking tests ${ }^{6,23,24}$. Metabolic syndrome, which comprises different cardiovascular risk factors, was associated with higher EDSS score at diagnosis, and with the risk of EDSS progression ${ }^{8}$. Another study combined different comorbidities by adding them one another, and showed a $200 \%$ increased risk of disability progression in subjects with at least 2 comorbidities ${ }^{6}$. Though some studies showed conflicting results ${ }^{25-27}$, in a meta-analysis, smoking was associated with higher average EDSS ${ }^{28}$, and, in a large British sample $(n=895)$, smokers were more at risk of reaching of milestone EDSS 4.0 and $6.0^{29}$. As such, the reaching of EDSS 6.0 is a proxy of increased risk of death, that could be at least in part mediated by cardiovascular risk factors, representing up to $17.3 \%$ causes of death ${ }^{22}$. Of note, cardiovascular comorbidities are associated with reduced survival more in MS, when compared with general population $11,30$.

The mechanisms by which cardiovascular risk factors affect MS remain unknown. First, even in the absence of overt cerebrovascular disease, hypercholesterolemia, diabetes and hypertension are associated with brain atrophy ${ }^{31,32}$, possibly due to increased peripheral inflammation ${ }^{33}$. Also, diabetes and other cardiovascular risk factors may increase the susceptibility to oxidative stress, change the blood vessel structure and function, and worsen the inflammatory response within the brain ${ }^{34}$. Not least, abnormalities of cerebral endothelial cells are reported in MS, and could be enhanced by cardiovascular risk factors ${ }^{35}$. As such, it is possible that multiple mechanisms contribute to explain interactions between multiple cardiovascular comorbidities and MS.

This article is protected by copyright. All rights reserved 
Age is part of Framingham risk score calculation, and is an important determinant of the associations with relapses, disability and DMT escalation (as shown by the significance of covariates in the statistical models). On the one hand, MS relapse rate declines with ageing, with younger individuals being more at risk of relapses than older subjects ${ }^{36}$. However, older individuals have an increased risk of vascular comorbidities, such as hypertension, hyperlipidaemia and diabetes, thus contributing to relapse risk. In our study, we have included age in both the Framingham risk score and the statistical models as a covariate; as such, we have mitigated the effect of age (and other not-modifiable risk factors, such as sex and disease duration) on the reaching of clinical outcomes in relation to the overal cardiovascular risk, measured with the Framingham risk score. For instance, notwithstanding the significant contribution of age, sex and disease duration, the Framingham risk score is an independent contributor to ARR, EDSS and DMT escalation. On the contrary, the association between older ager, higher cardiovascular risk and worse disability is more straightforward ${ }^{37,38}$.

We observed fewer cardiovascular events in our MS population (2.0\%), compared with expected rates estimated through Framingham risk score in the general population (3.0\%). MS patients have higher burden of cardiovascular comorbidities, when compared with general population ${ }^{11,39,40}$. As such, we cannot exclude that some cardiovascular events went missing in our retrospective study. However, the difference between expected and observed cardiovascular events also point towards an unsuitability of the Framingham risk score for predicting cardiovascular risk in MS. The Framingham risk score was originally developed in the general population and, thus, does not account for a number of factors which could affect the cardiovascular risk in MS (e.g., reduced mobility due to disability, use of steroids for relapses), with more specific scales deserving to be developed.

A number of limitations might have affected the present study. First, this is a retrospective study, hence less scientifically sound than a clinical trial or a prospective study. Looking at our population, patients were recruited in a single Italian MS centre and, so, the extent to which this sample is representative of the general MS population cannot be definitively assessed. Also, we only measured the cardiovascular risk at baseline, and did not asses changes over time; however, the Framingham risk score was specifically designed to predict 
the cardiovascular risk over the following years ${ }^{14}$. Unfortunately, standardized cholesterol measurements were not available for all subjects, and further studies are warranted on this issue, since an adverse lipid profile has been associated with MS evolution in several studies ${ }^{8,10}$; nevertheless, the Framingham risk score based on the BMI has showed similar capacity to predict the cardiovascular risk, when compared with the use of cholesterol levels ${ }^{14}$. Additional limitations could come from potential confounders, such as the use of multiple medications in patients with more comorbidities, with side effects or interactions influencing our outcomes. Some patients might have changed of DMT due to personal decision or to other factors which do not necessarily imply treatment escalation, though our results on treatment escalation are consistent with results on relapses. Cardiovascular comorbidities might also affect DMT response, deserving to be explored in future studies. Comorbid conditions are also associated with decreased quality of life in MS ${ }^{41}$, which we did not record in our study.

In conclusion, the Framingham risk score was associated with MS relapses, disability and treatment choices over 5 years, but is possibly not suitable for assessing the cardiovascular risk in MS. Correction and treatment of cardiovascular risk factors, along with lifestyle changes, should be carefully considered in the long-term management of MS.

\section{Funding}

The present study received no specific funding; laboratory and clinical variables were acquired with standard methods in the clinical practice.

\section{Declaration of conflicting interests}

Roberta Lanzillo has received honoraria from Almirall, Biogen, Merck, Teva, Roche, Novartis, Sanofi-Genzyme, and Teva. Antonio Carotenuto has received research grants from Almirall, and honoraria form Novartis and Roche.

Vincenzo Brescia Morra has received research grants from Italian MS Federation; and honoraria from Almirall, Bayer, Biogen, Merck, Roche, Novartis, Sanofi-Genzyme, and Teva. Marcello Moccia has received research grants from ECTRIMS-MAGNIMS, UK MS Society, and Merck; honoraria from Biogen, Merck, Novartis, and Roche; and consulting fees from Veterans Evaluation Services. 
Other authors have nothing to disclose.

\section{References}

1. Dobson R, Giovannoni G. Multiple sclerosis - a review. Eur J Neurol. 2019;26:27-40. doi:10.1111/ene.13819

2. Marrie RA. Comorbidity in multiple sclerosis: Past, present and future. Clin Invest Med. 2019;42(1):E5-E12. doi:10.25011/cim.v42i1.32383

3. Marrie RA, Reider N, Cohen J, Stuve O, Trojano M, Cutter G. A systematic review of the incidence and prevalence of cardiac, cerebrovascular, and peripheral vascular disease in multiple sclerosis. Mult Scler. 2015;21(3):318-331. doi:10.1177/1352458514564485

4. Moccia M, Lanzillo R, Palladino R, et al. The Framingham cardiovascular risk score in multiple sclerosis. Eur J Neurol. 2015;22(8):1176-1183. doi:10.1111/ene.12720

5. Kowalec K, McKay KA, Patten SB, et al. Comorbidity increases the risk of relapse in multiple sclerosis. Neurology. 2017;89(24):2455-2461. doi:10.1212/WNL.0000000000004716

6. Marrie RA, Rudick R, Horwitz R, et al. Vascular comorbidity is associated with more rapid disability progression in multiple sclerosis. Neurology. 2010;74(13):1041-1047. doi:10.1212/WNL.0b013e3181d6b125

7. Zhang $T$, Tremlett $H$, Leung $S$, et al. Examining the effects of comorbidities on diseasemodifying therapy use in multiple sclerosis. Neurology. 2016;86(14):1287-1295. doi:10.1212/WNL.0000000000002543

8. Sicras-Mainar A, Ruíz-Beato E, Navarro-Artieda R, Maurino J. Comorbidity and metabolic syndrome in patients with multiple sclerosis from Asturias and Catalonia, Spain. BMC Neurol. 2017;17(1):134. doi:10.1186/s12883-017-0914-2

9. Langer-Gould A, Brara SM, Beaber BE, Koebnick C. Childhood obesity and risk of pediatric multiple sclerosis and clinically isolated syndrome. Neurology. 2013;80(6):548-552. doi:10.1212/WNL.0b013e31828154f3

10. Tettey $\mathrm{P}$, Simpson S, Taylor B, et al. Adverse lipid pro fi le is not associated with relapse risk in MS: Results from an observational cohort study. J Neurol Sci. 2014;340(1-2):230-232. doi:10.1016/j.jns.2014.02.038

11. Palladino R, Marrie R, Majeed A, Chataway J. Evaluating the Risk of Macrovascular 
Events and Mortality Among People With Multiple Sclerosis in England. JAMA Neurol. 2020;77(7):1-9. doi:10.1001/jamaneurol.2020.0664

12. Chataway J, Schuerer N, Alsanousi A, et al. Eff ect of high-dose simvastatin on brain atrophy and disability in secondary progressive multiple sclerosis (MS-STAT): A randomised, placebo-controlled, phase 2 trial. Lancet. 2014;383(9936):2213-2221. doi:10.1016/S0140-6736(13)62242-4

13. Lanzillo R, Moccia M, Russo CV, et al. Therapeutic lag in reducing disability progression in relapsing-remitting multiple sclerosis: 8-year follow-up of two randomized add-on trials with atorvastatin. Mult Scler Relat Disord. 2019;28:193-196. doi:10.1016/j.msard.2018.12.042

14. D'Agostino RB, Vasan RS, Pencina MJ, et al. General cardiovascular risk profile for use in primary care: The Framingham heart study. Circulation. 2008;117(6):743-753. doi:10.1161/CIRCULATIONAHA.107.699579

15. European Medicine Agency (EMA). European public assessment reports. 2020. https://www.ema.europa.eu/en/medicines

16. Faul F, Erdfelder E, Lang AG, Buchner A. G*Power 3: A flexible statistical power analysis program for the social, behavioral, and biomedical sciences. Behav Res Methods. 2007;39(2):175-191. doi:10.3758/BF03193146

17. Palladino R, Vamos EP, Chang KCM, Khunti K, Majeed A, Millett C. Evaluation of the Diabetes Screening Component of a National Cardiovascular Risk Assessment Programme in England: a Retrospective Cohort Study. Sci Rep. 2020;10(1):1231. doi:10.1038/s41598-020-58033-3

18. Geraldes R, Juryńczyk M, Dos Passos G, et al. Distinct influence of different vascular risk factors on white matter brain lesions in multiple sclerosis. J Neurol Neurosurg Psychiatry. 2020;91(4):388-391. doi:10.1136/jnnp-2019-322369

19. Weinstock-Guttman B, Zivadinov R, Mahfooz N, et al. Serum lipid profiles are associated with disability and MRI outcomes in multiple sclerosis. J Neuroinflammation. 2011;8:127.

20. Laroni A, Signori A, Maniscalco GT, et al. Assessing association of comorbidities with treatment choice and persistence in MS. Neurology. 2017;89(22):2222-2229. doi:10.1212/WNL.0000000000004686

21. Moccia M, Palladino R, Lanzillo R, Triassi M, Brescia Morra V. Predictors of the 10- 
year direct costs for treating multiple sclerosis. Acta Neurol Scand. 2017;135(5):522528. doi:10.1111/ane.12630

22. Harding $\mathrm{K}$, Anderson $\mathrm{V}$, Williams $\mathrm{O}$, et al. A contemporary study of mortality in the multiple sclerosis population of south east Wales. Mult Scler Relat Disord. 2018;25:186-191. doi:10.1016/j.msard.2018.08.001

23. Fitzgerald KC, Salter A, Tyry T, Fox RJ, Cutter G, Marrie RA. Measures of general and abdominal obesity and disability severity in a large population of people with multiple sclerosis. Mult Scler. 2019. doi:10.1177/1352458519845836

24. Conway DS, Thompson NR, Cohen JA. Influence of hypertension, diabetes, hyperlipidemia, and obstructive lung disease on multiple sclerosis disease course. Mult Scler. 2017;23(2):277-285. doi:10.1177/1352458516650512

25. Lorefice L, Destro F, Fenu G, et al. The impact of modifiable risk factors on lesion burden in patients with early multiple sclerosis. Mult Scler Relat Disord. 2020;39:101886. doi:10.1016/j.msard.2019.101886

26. Hernán MA, Jick SS, Logroscino G, Olek MJ, Ascherio A, Jick H. Cigarette smoking and the progression of multiple sclerosis. Brain. 2005;128(Pt 6):1461-1465. doi:10.1093/brain/awh471

27. Koch $M$, Van Harten A, Uyttenboogaart $M$, De Keyser J. Cigarette smoking and progression in multiple sclerosis. Neurology. 2007;69(15):1515-1520. doi:10.1212/01.wnl.0000277658.78381.db

28. Heydarpour P, Manouchehrinia A, Beiki O, et al. Smoking and worsening disability in multiple sclerosis: A meta-analysis. Acta Neurol Scand. 2018;138(1):62-69. doi:10.1111/ane.12916

29. Manouchehrinia A, Tench CR, Maxted J, Bibani RH, Britton J, Constantinescu CS. Tobacco smoking and disability progression in multiple sclerosis: United Kingdom cohort study. Brain. 2013;136(Pt 7):2298-2304. doi:10.1093/brain/awt139

30. Chou IJ, Kuo CF, Tanasescu R, et al. Comorbidity in multiple sclerosis: its temporal relationships with disease onset and dose effect on mortality. Eur I Neurol. 2020;27:105-112. doi:10.1111/ene.14040

31. Enzinger C, Fazekas F, Matthews PM, et al. Risk factors for progression of brain atrophy in aging: Six-year follow-up of normal subjects. Neurology. 2005;64(10):17041711. doi:10.1212/01.WNL.0000161871.83614.BB

This article is protected by copyright. All rights reserved 
32. Knopman DS, Mosley TH, Catellier DJ, Sharrett AR. Cardiovascular risk factors and cerebral atrophy in a middle-aged cohort. Neurology. 2005;65(6):876-881. doi:10.1212/01.wnl.0000176074.09733.a8

33. Jefferson AL, Massaro JM, Wolf PA, et al. Inflammatory biomarkers are associated with total brain volume: The Framingham Heart Study. Neurology. 2007;68(13):10321038. doi:10.1212/01.wnl.0000257815.20548.df

34. Launer $\amalg$. Diabetes and brain aging: Epidemiologic evidence. Curr Diab Rep. 2005;5(1):59-63. doi:10.1007/s11892-005-0069-1

35. Minagar A, Jy W, Jimenez JJ, Alexander JS. Multiple sclerosis as a vascular disease. Neurol Res. 2006;28(3):230-235. doi:10.1179/016164106X98080

36. Tremlett $\mathrm{H}$, Zhao $\mathrm{Y}$, Joseph J, et al. Relapses in multiple sclerosis are age- and timedependent. J Neurol Neurosurg Psychiatry. 2008;79(12):1368-1374. doi:10.1136/jnnp.2008.145805

37. Scalfari A. MS progression is predominantly driven by age-related mechanisms - YES. Mult Scler. 2019;25(7):902-904. doi:10.1177/1352458518820633

38. Weideman AM, Tapia-Maltos MA, Johnson K, Greenwood M, Bielekova B. Metaanalysis of the age-dependent efficacy of multiple sclerosis treatments. Front Neurol. 2017;8:577. doi:10.3389/fneur.2017.00577

39. Persson $\mathrm{R}$, Lee $\mathrm{S}$, Yood $\mathrm{MU}$, et al. Incident cardiovascular disease in patients diagnosed with multiple sclerosis: A multi-database study. Mult Scler Relat Disord. 2020;37:101423. doi:10.1016/j.msard.2019.101423

40. Smith KA, Burkill S, Hiyoshi A, et al. Comorbid disease burden among MS patients 1968-2012: A Swedish register-based cohort study. Mult Scler. 2020. doi:10.1177/1352458520910497

41. Berrigan LI, Fisk JD, Patten SB, et al. Health-related quality of life in multiple sclerosis: Direct and indirect effects of comorbidity. Neurology. 2016;86(15):1417-1424. doi:10.1212/WNL.0000000000002564 
Table 1. Baseline demographics and clinical features.

\begin{tabular}{lc}
\hline & $\begin{array}{c}\text { MS patients } \\
(n=251)\end{array}$ \\
\hline Age, years & $42.1 \pm 11.3$ \\
Sex, female & $154(61.3 \%)$ \\
Disease duration, years & $8.1 \pm 6.3$ \\
EDSS & $3.5(1.0-7.5)$ \\
Smokers & $96(38.2 \%)$ \\
BMI, $\mathrm{kg} / \mathrm{m}^{2}$ & $25.4 \pm 4.5$ \\
Systolic blood pressure, $\mathrm{mmHg}$ & $119.1 \pm 13.9$ \\
Use of hypertensive medications & $33(13.1 \%)$ \\
Diabetes & $5(2.0 \%)$ \\
Framingham risk score & $6.5 \pm 6.6 \%$
\end{tabular}

Table shows demographics, cardiovascular, and MS clinical features at baseline. 
Table 2. Follow-up MS clinical features.

\begin{tabular}{|c|c|c|c|c|c|}
\hline & & \multirow[t]{2}{*}{ Coeff } & \multicolumn{2}{|c|}{$95 \% \mathrm{Cl}$} & \multirow[t]{2}{*}{ P-value } \\
\hline & & & Lower & Upper & \\
\hline ARR & $0.07 \pm 0.16$ & 0.43 & 0.18 & 0.59 & $<0.01^{*}$ \\
\hline \multirow[t]{3}{*}{ EDSS } & $3.5(1.0-8.0)$ & 2.72 & 2.50 & 2.94 & $<0.01 *$ \\
\hline & & HR & \multicolumn{2}{|c|}{$95 \% \mathrm{Cl}$} & P-value \\
\hline & & & Lower & Upper & \\
\hline Relapse occurrence & $52 / 251(20.7 \%)$ & 1.31 & 1.03 & 1.68 & $0.02 *$ \\
\hline Time to relapse, months & $14.5 \pm 13.0$ & & & & \\
\hline Reaching of EDSS $6.0^{\# \#}$ & $13 / 217(6.0 \%)$ & 1.19 & 1.05 & 3.01 & $0.02^{*}$ \\
\hline Time to EDSS 6.0, months & $23.5 \pm 17.2$ & & & & \\
\hline DMT escalation ${ }^{\#}$ & $60 / 135$ (44.4\%) & 1.62 & 1.22 & 3.01 & $0.03^{*}$ \\
\hline Time to DMT escalation, months & $43.1 \pm 20.1$ & & & & \\
\hline
\end{tabular}

Table shows MS clinical feature during follow-up. Coefficients (Coeff), hazard ratios (HR), 95\% Confidence Intervals $(95 \% \mathrm{Cl})$, and $\mathrm{p}$-values are presented from Poisson regression (ARR), ordered logistic regression model (EDSS), and Cox proportial hazards regression models (time to relapse, time to EDSS 6.0, and time to DMT escalation), as appropriate (* indicates $\mathrm{p}<0.05)$.

\#Among all patients ( $n=251)$.

\#\#Among patients with baseline EDSS<6.0 ( $n=217)$. 
Table 3. DMTs.

\begin{tabular}{lcc}
\hline & $\begin{array}{c}\text { Baseline } \\
(2014)\end{array}$ & $\begin{array}{c}\text { Follow-up } \\
\text { (up to 2020) }\end{array}$ \\
\hline Alemtuzumab & - & $16(6.4 \%)$ \\
Dimethyl-fumarate & $5(2.0 \%)$ & $23(9.2 \%)$ \\
Fingolimod & $37(14.7 \%)$ & $52(20.7 \%)$ \\
Interferon-beta, Glatiramer Acetate & $141(56.2 \%)$ & $55(21.9 \%)$ \\
Natalizumab & $54(21.5 \%)$ & $33(13.1 \%)$ \\
Ocrelizumab, Rituximab & - & $20(8.0 \%)$ \\
Teriflunomide & $5(2.0 \%)$ & $16(6.4 \%)$ \\
Untreated & $9(3.6 \%)$ & $36(14.3 \%)$ \\
\hline
\end{tabular}

Table shows number and percent of patients receiving different DMTs at baseline and follow-up. 

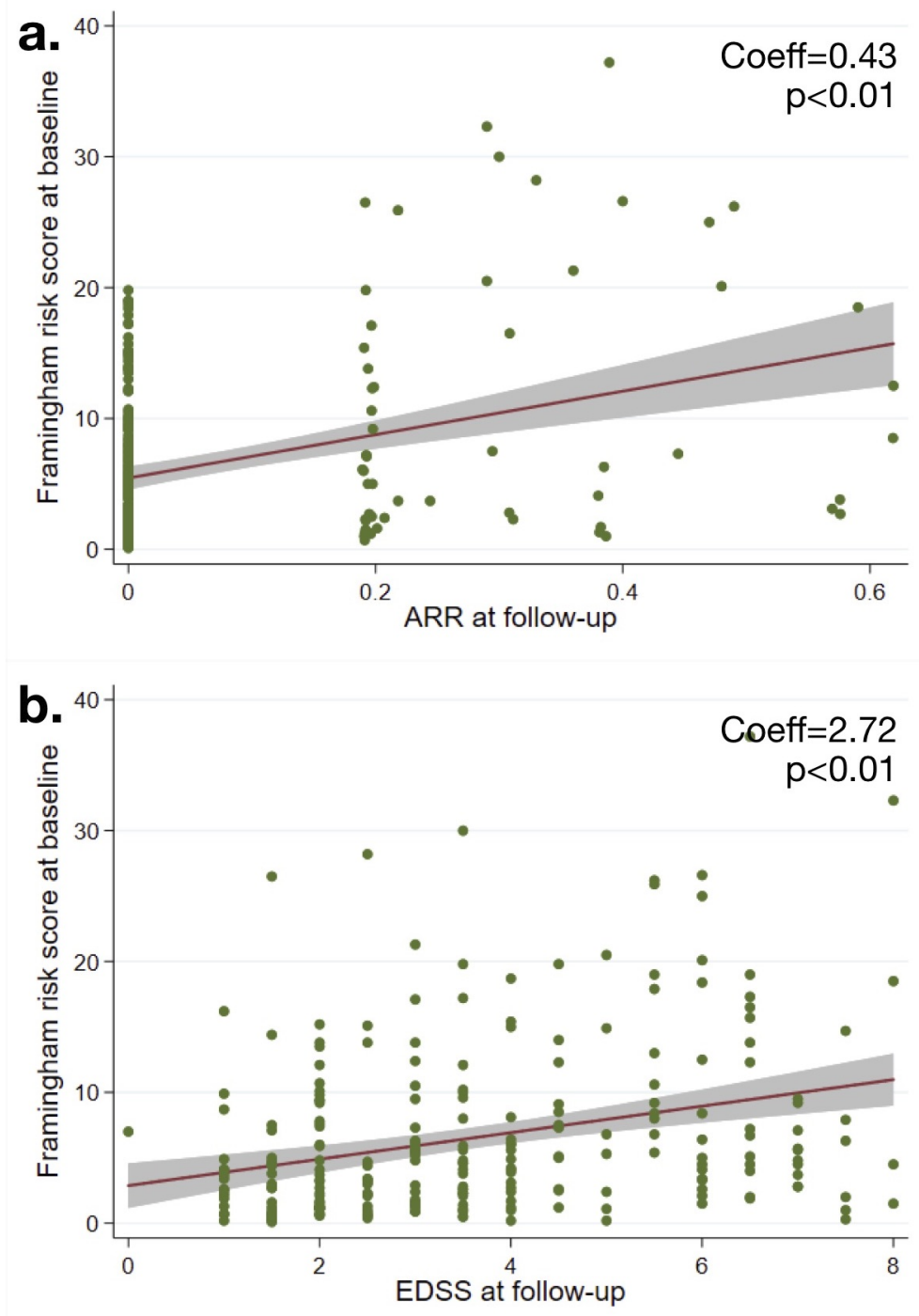

ene_14608_f1.jpg

This article is protected by copyright. All rights reserved 

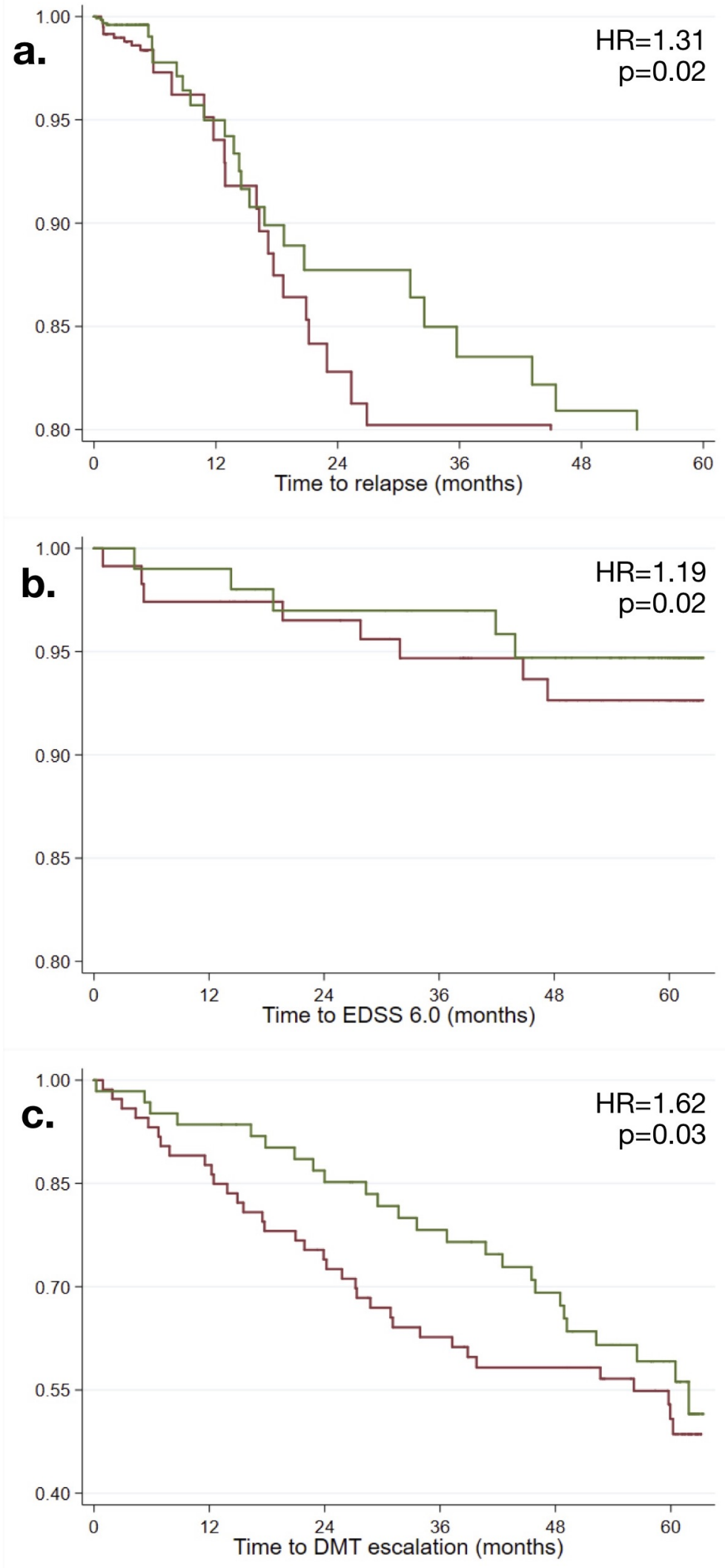

ene_14608_f2.jpg 